Elsevier Editorial System(tm) for Polymer Manuscript Draft

Manuscript Number: POLYMER-18-1610

Title: Looking for the Simplicity in Polymer Networks - Structure Changes and Comparative Analysis of Theoretical Approaches to Deformation of Semi-Crystalline Polyolefines

Article Type: Research Paper

Section/Category: Polymer Physics and Physical Chemistry

Keywords: Polyolefines; Slip-link model; Polymer networks.

Corresponding Author: Dr. Maxim A. Shcherbina, Dr.

Corresponding Author's Institution: Enikilopov Institute of Synthetic Polymer Materials RAS

First Author: Sergei N Chvalun, Professor

Order of Authors: Sergei N Chvalun, Professor; Yaroslav I Odarchenko, Dr.; Meshchankina Y Marina; Maxim A. Shcherbina, Dr.; Natalia P Bessonova, Dr.; Rudy Deblieck, Dr; Mark Boerakker; Klaas Remerie, Dr; Litvinov Viktor, Dr.

Abstract: To establish relationships between the molecular structure of polyolefines and their physical characteristics which determine possible commercial applications, structural changes and tensile deformation response up to deformations beyond the natural draw ratio were investigated using a variety of experimental approaches. True stressstrain curves were measured at different temperatures so as to estimate the available effective network density, which will eventually define the failure mode of the material under investigation. Analysis of the deformation by means of tensile strain hardening, assuming the HawardThackray spring dashpot decoupling assumption by means of Edward-Vilgis' non-Gaussian rubber-elastic slip-link model, reveals the role of transient and fixed network nodes.

It was established by differential scanning calorimetry and X-ray diffraction analysis that the transformation from lamellar to fibrillar morphology passes through the several pronounced stages: deformation of initial lamellae $(\square<1.5)$; destruction of lamellar structure through the tilt; slippage of molecules in the crystallites; simultaneous formation of fibrils with structural characteristics depending on the molecular structure and on deformation conditions; deformation of the formed fibrillar structure; tilting - formation of chevrons for high molecular weight low density polyethylene or slippage of fibrils and void formation.

Distinction between fixed and transient slip link network contributions reveals neatly that although there is a slight drop in the fixed link network density with increasing temperature, this contribution remains of the same order of magnitude and predominantly related to the molecular mass. This observation enhances the idea that the network of entanglements that remain fixed on the time scale of the measurement is actually entropic in nature and does not depend greatly on temperature. 
Considering slip link contributions, one can find them to be prominently present at ambient temperature and their importance becomes negligible at elevated temperature, except for the case of the most crystalline material. 


\title{
Looking for the Simplicity in Polymer Networks - Structure Changes and Comparative Analysis of Theoretical Approaches to Deformation of Semi- Crystalline c
}

\author{
Chvalun S.N. ${ }^{\mathrm{a}, \mathrm{b}}$, Odarchenko Ya.I. ${ }^{\mathrm{a}}$, Meshchankina M.Yu. ${ }^{\mathrm{b}}$, \\ Shcherbina M.A. ${ }^{\text {b.c }}$, Bessonova N.P. ${ }^{a}$, Deblieck R.A.C. ${ }^{\text {, }}$, \\ Boerakker M. ${ }^{\mathrm{d}}$, Remerie K. ${ }^{\mathrm{e}}$, Litvinov V. ${ }^{\mathrm{f}}$ \\ ${ }^{a}$ Enikolopov Institute of Synthetic Polymer Materials RAS, $70 \mathrm{ul}$. \\ Profsoyuznaya, 117393 Moscow, Russian Federation. \\ b National Research Centre "Kurchatov Institute", 1 pl. Academika \\ Kurchatova, 123098 Moscow, Russian Federation. \\ ${ }^{\mathrm{c}}$ Moscow Institute of Physics and Technology, 4 Institutsky line, \\ 141700 Dolgoprudny, Moscow region, Russian Federation. \\ ${ }^{\mathrm{a}}$ Sabic Technology and Innovation, \\ PO Box 475, 6160 AL Geleen, Geleen, the Netherlands \\ ${ }^{\mathrm{e}}$ Faculty of Science and Engineering, University of Groningen, \\ PO Box 72, 9700 AB Groningen, the Netherlands \\ ${ }^{\mathrm{f}}$ V. Lit. Consult, Gozewijnstraat 4, 6191 WV, Beek, The Netherlands.
}

\begin{abstract}
To establish relationships between the molecular structure of polyolefines and their physical characteristics which determine possible commercial applications, structural changes and tensile deformation response up to deformations beyond the natural draw ratio were investigated using a variety of experimental approaches. True stress-strain curves were measured at different temperatures so as to estimate the available effective network density, which will eventually define the failure mode of the material under investigation. Analysis of the deformation by means of tensile strain hardening, assuming the Haward-Thackray spring dashpot decoupling assumption by means of Edward-Vilgis' non-Gaussian rubber-elastic slip-link model, reveals the role of transient and fixed network nodes.

It was established by differential scanning calorimetry and X-ray diffraction analysis that the transformation from lamellar to fibrillar morphology passes through the several pronounced stages: deformation of initial lamellae $(\lambda<1.5)$; destruction of lamellar structure through the tilt; slippage of molecules in the crystallites; simultaneous formation of fibrils with structural characteristics depending on the molecular structure and on deformation conditions; deformation of the formed fibrillar structure; tilting formation of chevrons for high molecular weight low density polyethylene or slippage of fibrils and void formation.

Distinction between fixed and transient slip link network contributions reveals neatly that although there is a slight drop in the fixed link network density with increasing temperature, this contribution remains
\end{abstract}


of the same order of magnitude and predominantly related to the molecular mass. This observation enhances the idea that the network of entanglements that remain fixed on the time scale of the measurement is actually entropic in nature and does not depend greatly on temperature. Considering slip link contributions, one can find them to be prominently present at ambient temperature and their importance becomes negligible at elevated temperature, except for the case of the most crystalline material. 


\section{Introduction}

Nanoscale microphase separated systems attract a great attention in both academic and applied research circles in last decades[1,2,3]. Recent achievements in the chemistry of polyolefines stipulated by the progress in the design of new catalytic systems, allow synthesis of polymers with highly defined molecular structure, molecular weight distribution, and correspondingly with well-defined supramolecular organization, degree of crystallinity and a wide range of adjustable properties - from rubber-like elastomers to highly crystalline thermoplastics. Low crystalline materials with the crystallinity less than $10 \%$ exhibit pronounced ability to large reversible deformations while highly crystalline ones are able for only limited deformations because in such systems deformation behavior is determined by the changes in crystalline lamellae. Thus, a unique opportunity to adjust material properties lies in variation of polymer crystallinity: with its increase formation of continuous crystalline phase (percolation) leads to the switch from typical rubber-like behaviour characterized by large, uniform, reversible deformations, to the typical cold drawing when necking occurs.

Changes in the molecular structure of copolymers lead via a complex crystallization behavior to significantly different properties. Numerous efforts were devoted to large deformations of such microheterogeneous polymer systems $[4,5,6,7,8,9]$, and to the establishment of relationships between the chemical structure of polyolefins and their mechanical properties determining possible commercial applications. Amongst the most important properties, besides modulus, is toughness on short and long time scales, think about the creep failure resistance of pipe grade materials.

Understanding the strain hardening response is crucial and central in understanding this failure resistance[10] as it determines the surface energy that is the central physical observable in the balance between craze propagation and ductile failure[10,12].

Various rubber elastic models may be used to describe the strain hardening behavior of semicrystalline polymers. Recent developments in the interpretation of mechanical behavior of thermoplastics have increasingly employed the concept of the polymer chain conformation[11,12,13,14,15]. In its application to solid rubbery materials, it is common to employ the hypothesis of Haward and Thackray[15] that elastic and viscous forces may be modelled in parallel. This assumption makes it possible to represent not only deformations measured in tension, but also those resulting from the application of other types of load such as compression and shear.

From experimental point of view, powerful combination of deformation calorimetry[16,17] and wide and small angle $\mathrm{X}$-ray analysis allows to establish direct links between the molecular response on deformation and changes at the macroscopic level. Based on these data it is possible to elucidate important aspects of the mechanical behaviour of polymer systems such as energy contribution to the elasticity of a rubber matrix, limited chain extensibility, reversibility of deformation, hysteresis phenomena during cyclic deformation, and the role of hard domains in the process of high elongation. It 
is also possible to distinguish the phase transitions, to study strain-induced crystallization and to study the nature of cross-link networks.

In the present study special attention is paid to the deformation mechanisms of polyolefines of different chemical structure at elevated temperatures when structural rearrangements of crystalline blocks proceed more easily. The strain hardening is analysed by means of, Neo-Hookean, Mooney-Rivlin and Edward-Vilgis' non-Gaussian slip-link model, in order to estimate the nature of the available molecular and transient crystalline network.

\section{Characteristics of PE samples}

Four PE samples with different molecular masses and densities were studied. The density is controlled by the composition of the polymer, i.e., a number of chain branches hindering crystallization:

1. HDPE (density 0.964; MFI = 8), hereinafter PE-964;

2. bimodal HDPE (density 0.949; MFI = 0.3), hereinafter PE-949;

3. LLDPE-926 (density 0.926; MFI = 0.7), hereinafter PE-926;

4. LLDPE-918 (density 0.918; MFI = 1.0), hereinafter PE-918.

We have to emphasize that melt flow index varies substantially, as the samples are characterized by different molecular masses. This fact should be taken into account in our further considerations. The films were prepared by compression molding of pellets at high temperature with subsequent cooling to room temperature. The materials were pressed at $160^{\circ} \mathrm{C}$ to a uniform thickness sheet using the following procedure: $5 \mathrm{~min}$ of heating at $0 \mathrm{kN}$ load, $3 \mathrm{~min}$ at $10 \mathrm{kN}$ load, $3 \mathrm{~min}$ at $50 \mathrm{kN}$ load and cooling down to room temperature at a load of $180 \mathrm{kN}$. After pressing, the samples were annealed for $\mathrm{l} \mathrm{h}$ at $120^{\circ} \mathrm{C}$ and then slowly cooled down to a room temperature by switching off the chamber temperature. Finally, the test specimens (ISO37 type 3 - 1994 (E)) were punched from the pressed sheets. To elucidate an effect of preparation conditions on crystallinity and thermal behavior of the original pellets, two different types of samples were studied: 1) films quenched in a mixture of water and ice; 2) slowly crystallized films.

DSC measurements were performed using Mettler DSC-30 and PerkinElmer DSC -7 instruments. As-prepared films of $2 \div 10 \mathrm{mg}$ weight were heated from $-150^{\circ} \mathrm{C}$ to $+250^{\circ} \mathrm{C}$ with the rate of $20^{\circ} \mathrm{C} / \mathrm{min}$. Degree of crystallinity was calculated as a ratio of the heat of fusion of given sample to that of an ideal PE $\operatorname{crystal}\left(\Delta H_{0}=290 \mathrm{~J} / \mathrm{g}\right)$.

Detailed characterization of the structure of as-prepared PE films and of its transformation in the process of deformation was carried out by X-ray scattering analysis. Wide angle $\mathrm{X}$-ray 2D diffraction patterns were obtained using a flat X-ray camera (Ni-filtered $\mathrm{CuK}_{\alpha}$ radiation), the spot size of the focused beam on the sample being ca. $300 \mu \mathrm{m}$ in diameter. Linear equatorial and meridian diffraction scans of drawn fibers were recorded in the transmission mode with the use of a DRON-3 instrument $\left(\mathrm{CuK}_{\alpha}\right.$ radiation, curved quartz crystal monochromator). Degree of crystallinity of as-received 
samples was estimated from wide angle data as a ratio of the integral intensity of crystalline diffraction peaks to the total scattering, after the subtraction of instrumental background and correction for Lorentz and polarization factors. The lateral and longitudinal crystallite sizes were obtained from the integral half-widths of corresponding reflections using the Scherrer equation after correction for instrumental broadening. Structural changes on supramolecular hierarchical level in the process of sample deformation were monitored by small angle X-ray scattering using the compact Kratky camera (Ni-filtered $\mathrm{CuK}_{\alpha}$ radiation). Long period of microfibrillar structure was calculated with the use of Bragg equation after application of the desmearing procedure by SYRENA program. Moreover, small angle 2D X-ray patterns were also recorded by a pinhole camera with high angular resolution.

As-prepared films appeared to be semi-crystalline materials. Their WAXS patterns (Figure la) reveal the presence of broad amorphous halo as well as of strong 110 and 200 reflections characteristic for the orthorhombic crystal lattice of PE. Degree of crystallinity estimated as a ratio of the integral intensity of diffraction peaks to the total scattering, as well as lateral sizes of crystallites, are symbate to the macroscopic density of the samples (see Table 1). They are also in a good agreement with the data of differential scanning calorimetry. Moreover, one can observe an intensive maximum on small-angle X-Ray diffractograms of both HDPE and LLDPE samples (Figure $\mathrm{lb}$ ). The values of corresponding long periods are also shown in Table l. The intensity of the SAXS peaks is rather lower for LLDPE films compared to that in HDPE probably due to a structure factor effect, that is lamellar persistence which is lower in branched materials.
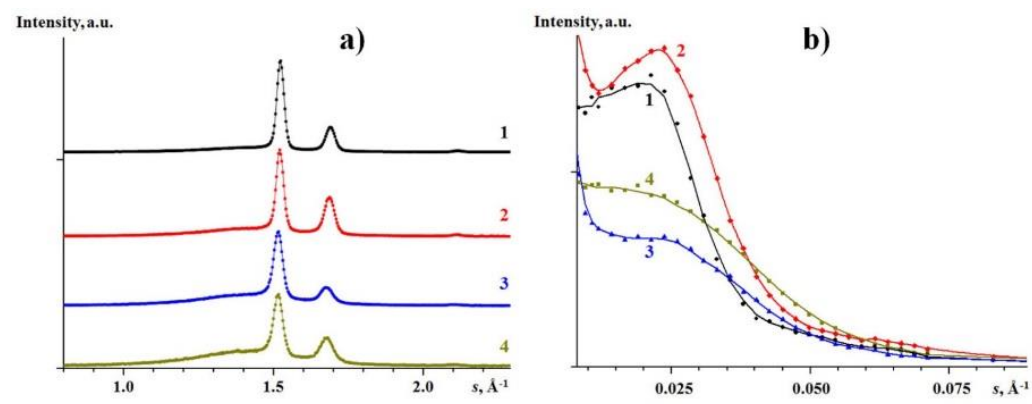

Figure 1: Wide- (a) and small-angle (b) X-Ray patterns of as-prepared films of PE-964 (1), PE-949 (2), PE-926 (3) and PE-918 (4).

Thermal behaviour of PE depends substantially on the macroscopic density of material as well as on the deformation conditions such as draw ratio and temperature. Figure 2 representing DSC thermograms of the first heating of as-prepared films and highly drawn fibres of PE-918, PE-926, PE-949 and PE-964 oriented at $T_{\text {def }}=70^{\circ} \mathrm{C}$ serves as an example of such statement. All thermographs reveal the endothermal peaks corresponding to the melting PE crystals in the temperature range of $80 \div 135^{\circ} \mathrm{C}$. Subsequent cooling is 
accompanied by exothermic effects of crystallization. Figure 3 summarizes the draw ratio dependencies of the melting temperature and of the heat of fusion for PE samples of different structure. One can easily see that for samples oriented both at $20^{\circ} \mathrm{C}$ and $70^{\circ} \mathrm{C}$, the density of the material directly correlates to its melting temperature at any given draw ratio.

Table l. Structural and thermal characteristics of as-prepared films*

\begin{tabular}{|c|c|c|c|c|c|c|c|c|}
\hline $\begin{array}{c}\text { Sample } \\
\text { (density) }\end{array}$ & $T_{\mathrm{m}}{ }^{\circ} \mathrm{C}$ & $\begin{array}{c}\Delta H_{\mathrm{m}}, \\
\mathrm{J} / \mathrm{g}\end{array}$ & $\varphi_{\text {DSC }}$ & $T_{\mathrm{cr}}{ }^{\circ} \mathrm{C}$ & $\begin{array}{c}\Delta \mathrm{Hcr}, \\
\mathrm{J} / \mathrm{g}\end{array}$ & $\begin{array}{c}\varphi \\
\text { X-Ray }\end{array}$ & $\begin{array}{c}\mathrm{l}_{110}, \\
\mathrm{~nm}\end{array}$ & $\begin{array}{c}\mathrm{L}, \\
\mathrm{nm}\end{array}$ \\
\hline PE-918 & 120 & 96 & 0.33 & 106 & 82 & 0.35 & 20 & 22 \\
\hline PE-926 & 120 & 136 & 0.47 & 107 & 129 & 0.46 & 23 & 22 \\
\hline PE-949 & 127 & 142 & 0.49 & 114 & 157 & 0.56 & 33 & 25 \\
\hline PE-964 & 135 & 211 & 0.73 & 115 & 212 & 0.67 & 40 & 28 \\
\hline
\end{tabular}

$* T_{\mathrm{m}}$ is the melting temperature obtained as a maximum of corresponding endothermal peak on the DSC thermogram; $\Delta H_{\mathrm{m}}$ is melting heat; $\varphi_{\mathrm{DSC}}$ is degree of crystallinity calculated using DSC; $T_{\mathrm{cr}}$ is the crystallization temperature obtained as a maximum of corresponding exothermal peak; $\Delta H_{\mathrm{cr}}$ is crystallization heat; $\varphi_{\mathrm{X} \text {-Ray }}$ is degree of crystallinity calculated using WAXS data; $l_{110}$ is lateral crystallite size calculated from the half-width of 110 reflection; $L$ is the long period.
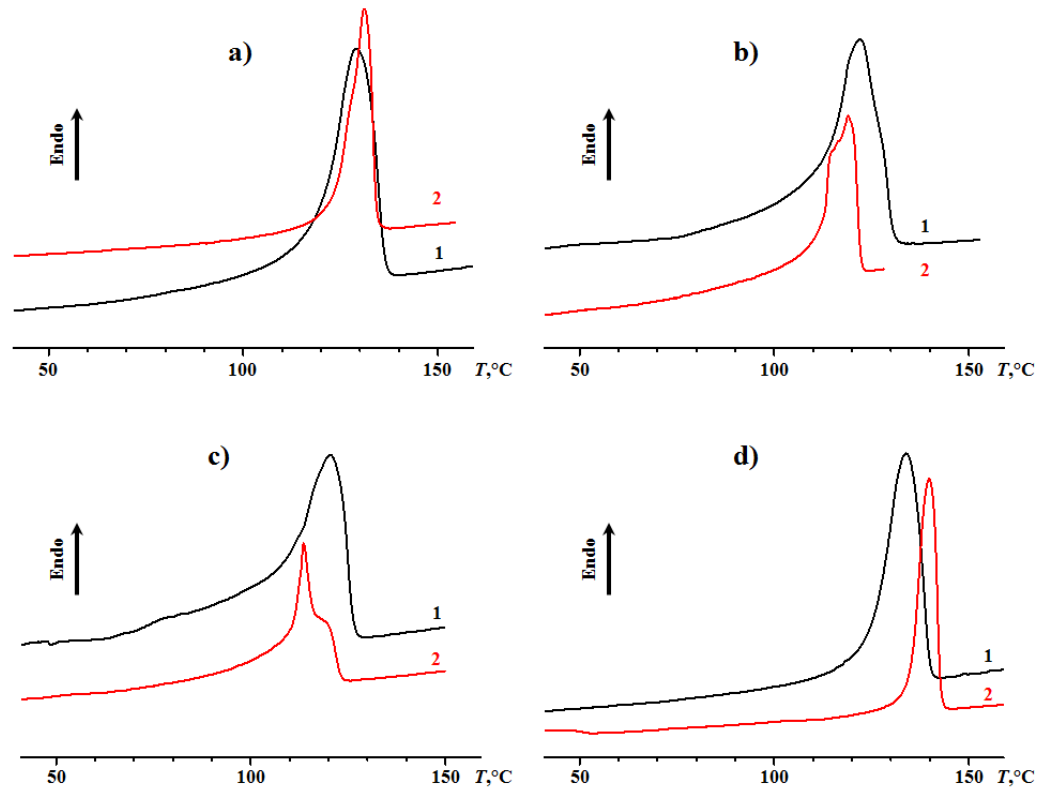

Figure 2: DSC thermograms of the first heating of as-prepared films (1) and highly oriented fibers (2) of PE-918 (a), PE-926 (b), PE-949 (c) and PE-964 (d) drawn at $70^{\circ} \mathrm{C}(2)$. The draw ratio of the fibers is $\lambda=8.5 ; 8.0 ; 7.0 ; 28$ on figures a-d correspondingly.

At the initial stages of deformation, crystallinity of all samples decreases and then starts to increase reaching the value of $80 \%$ in case of HDPE for both deformation temperatures. The degree of crystallinity of the other samples are lower but show the same tendency. Such behaviour of $K_{\Delta \mathrm{H}}(\lambda)$ can be explained by the deformation of virgin lamella $(\lambda=1 \div 2)$, their 
break-up and simultaneous formation of a new fibrillar morphology $(\lambda=1.5 \div 4)$, and finally by the further extension of the fibrils $(\lambda>6)$. Of course, the ranges of strain for these processes vary substantially for the studied polymers. The rise of calculated crystallinity with the draw ratio on the latter stage can be explained by the increasing contribution of bundles of tie molecules to the total heat of fusion[18,19,20].
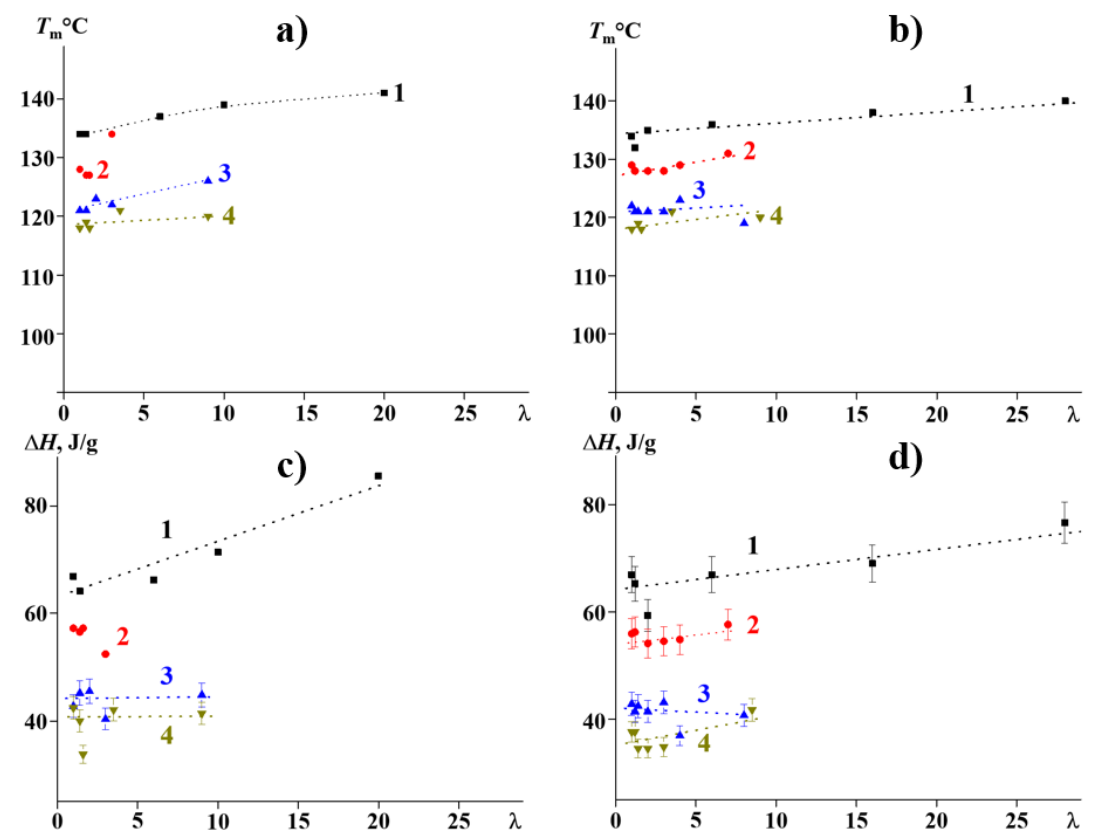

Figure 3: Draw ratio dependencies of melting temperature $(a, b)$ and heat of fusion (c,d) for the samples of PE-964 (1), PE-949 (2), PE-926 (3) and PE-918 (4) oriented at $20^{\circ} \mathrm{C}(\mathrm{a}, \mathrm{c})$ and $70^{\circ} \mathrm{C}(\mathrm{b}, \mathrm{d})$.

Analysis of the X-Ray data obtained in the process of deformation of PE samples with different molecular structure (Figures 4-7) confirms the proposed model. Although the character of structural changes is similar for the different orientation temperatures, only the patterns obtained at $T_{\text {def }}=70^{\circ} \mathrm{C}$ are shown. The character of such changes is typical for the crystalline РЕ[Ошибка! Закладка не определена.,Ошибка! Закладка не определена.]. In zone $1(1<\lambda<1.2-1.4)$ deformation of virgin lamellae takes place: the lamellae perpendicular to drawing orientation move away from each other, the parallel lamellae remain intact and the rest of them concentrates at an angle of $45^{\circ}$. On further orientation, at $\lambda>1.2$, crystallites re-orient themselves according to the Peterlin scheme[21], so as their crystallographic axis $\mathrm{a}$ is almost perpendicular to the drawing direction, thus 110 and 020 reflections form four-point azimuthal distribution, sometimes not uniform. On this stage the lamellae, especially those oriented at $45^{\circ}$ to the deformation direction, start to fragment through the tilt and slippage of chain segments, transforming into the fibrillar structure with the $c$-axis parallel to the draw 
direction. This transformation is accompanied by an initial decrease in crystallinity as can be seen on Figure 3.

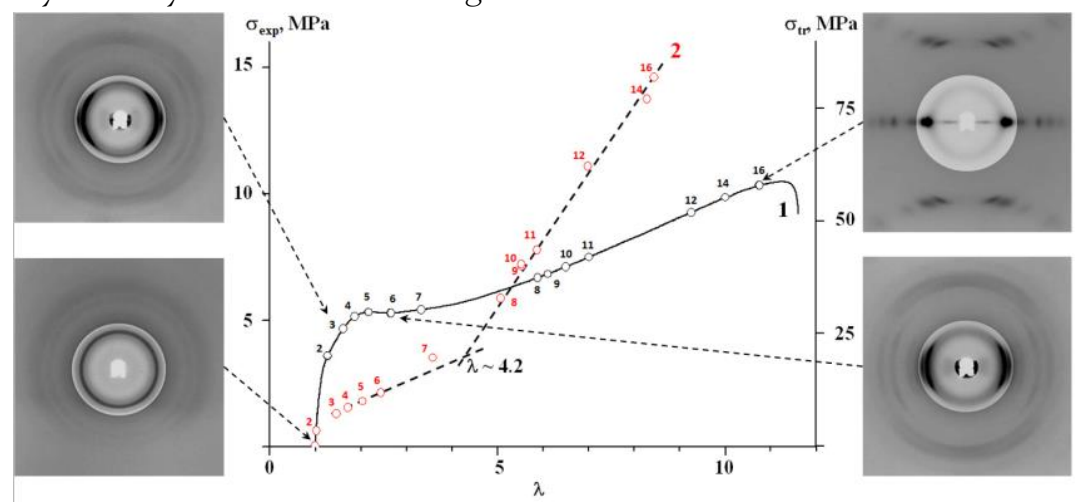

Figure 4: Deformation induced changes in 2-D WAXS patterns of PE-918 samples oriented at $T_{\mathrm{dr}}=70^{\circ} \mathrm{C} .1$ - experimental stress-strain curve, large circles show the points used for the calculation of true stress - true strain dependence (2).
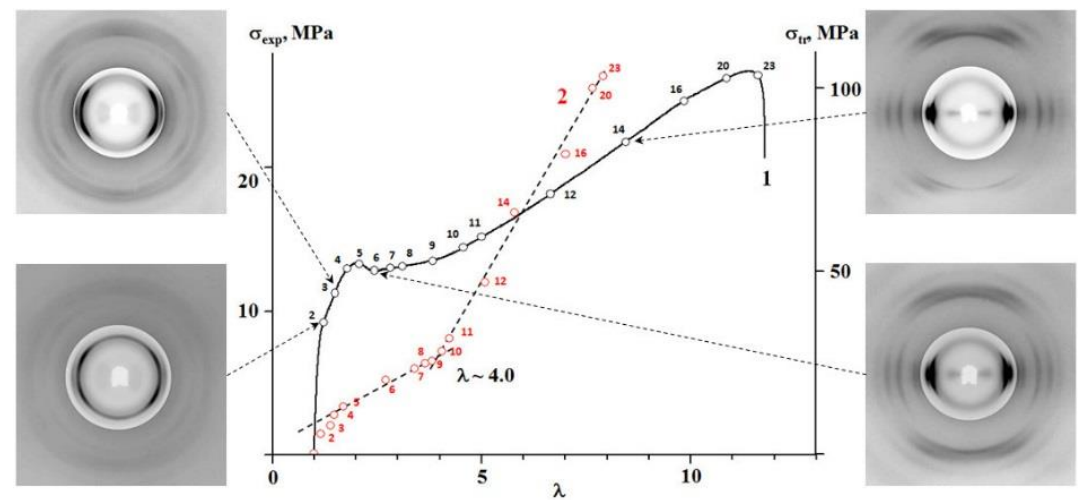

Figure 5: Deformation induced changes in 2-D WAXS patterns of PE-926 samples oriented at $T_{\mathrm{dr}}=70^{\circ} \mathrm{C} .1$ - experimental stress-strain curve, large circles show the points used for the calculation of true stress - true strain dependence (2).
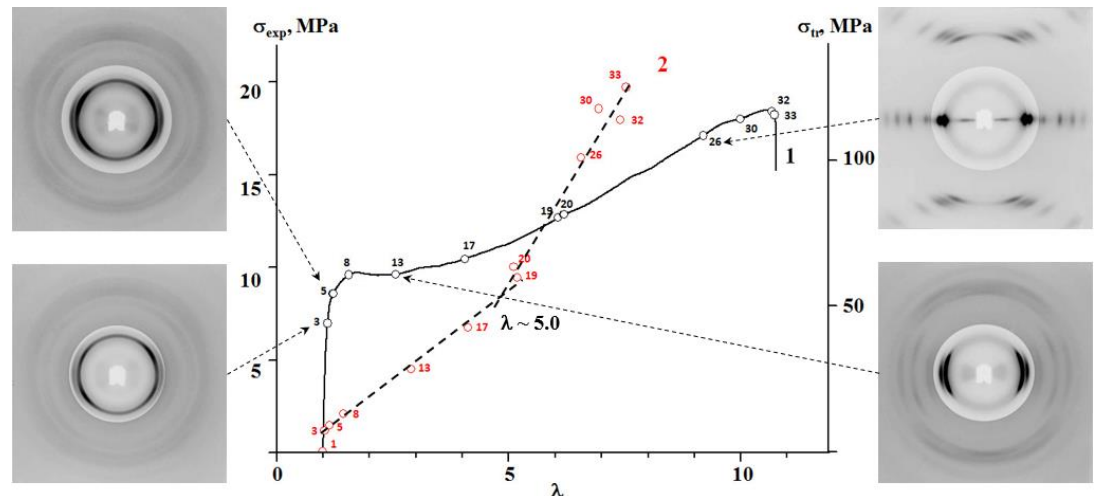
Figure 6: Deformation induced changes in 2-D WAXS patterns of PE-949 samples oriented at $T_{\mathrm{dr}}=70^{\circ} \mathrm{C}$. 1 - experimental stress-strain curve, large circles show the points used for the calculation of true stress - true strain dependence (2).

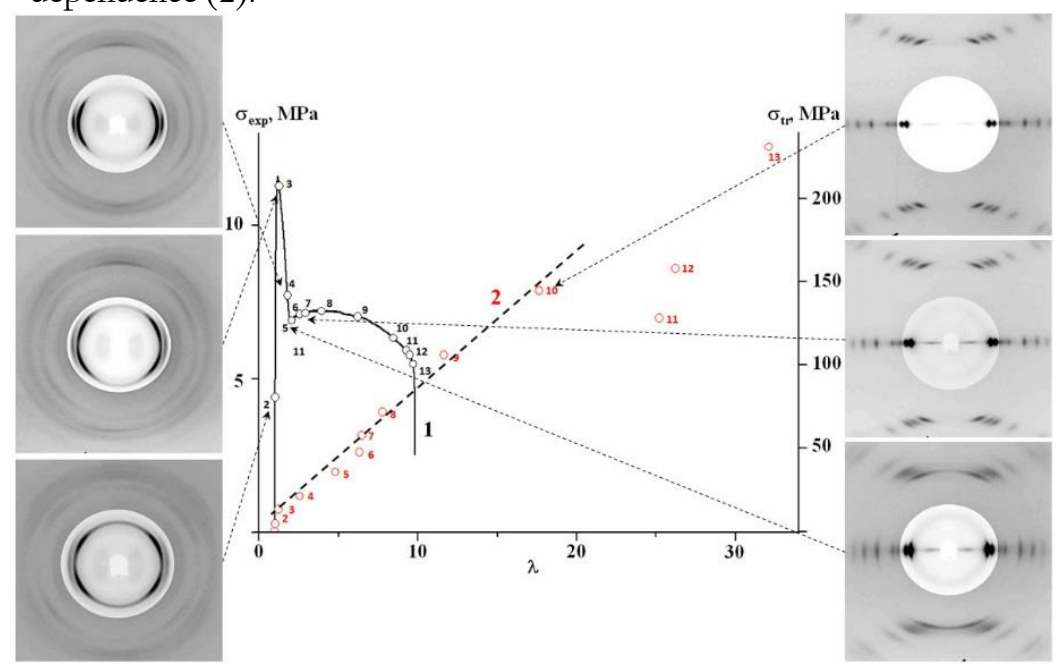

Figure 7: Deformation induced changes in 2-D WAXS patterns of PE-964 samples oriented at $T_{\mathrm{dr}}=70^{\circ} \mathrm{C} .1$ - experimental stress-strain curve, large circles show the points used for the calculation of true stress - true strain dependence (2)

Average longitudinal crystallite size $l_{002}$ in oriented fibers is smaller than that in the as-received samples and is equal to $13 \div 17 \mathrm{~nm}$ depending on the molecular structure of PE. Usually it remains constant after neck propagation, with the only exception being PE-964 where $l_{002}$ increases continuously up to $25 \mathrm{~nm}$. Average lateral crystallite size $l_{110}$ decreases in the process of orientation from 22 to $8 \mathrm{~nm}$ for LLDPE and from 33 to $14 \mathrm{~nm}$ for HDPE.

SAXS patterns of oriented samples of PE-926 reveal (Figure 8) that deformation to $\lambda=1.2$ is accompanied by the development of four-point streak-like scattering protruding from the origin; simultaneously the oval scattering from the lamellae becomes weaker. At $\lambda=1.4$ the streak scattering is getting stronger and new meridional maxima appear. The increase of draw ratio up to $\lambda=4$ results in the disappearance of streaks, only meridional scattering can be observed. On further deformation its intensity decreases and the shape changes from four-point one. Such type of SAXS pattern is characteristic for chevron-like supramolecular organization in which fibrillar crystallites have their face surfaces tilted with the respect to the direction of orientation. Supposedly, highly dense network of entanglements in interfibrillar amorphous regions prevents the deformation of fibrils and leads to the tilting of interface.

Thus, thermal and structural studies of PE samples of different density allowed us to establish relations between the deformation and the structure changes of the studied samples. The next step will be to relate such changes to the developed theoretical models of polymer deformation. 
a)

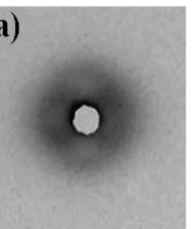

b)
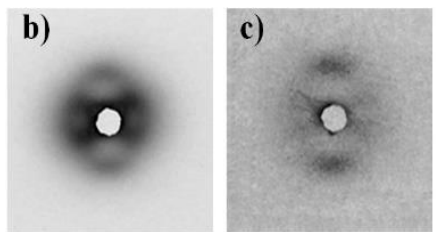

d)

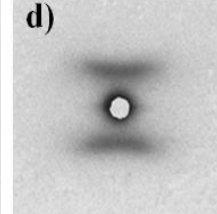

e)

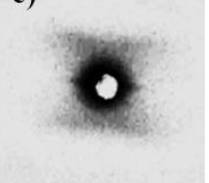

Figure 8: Deformation induced changes in 2-D SAXS patterns of PE-926 samples oriented at $T_{\mathrm{dr}}=70^{\circ} \mathrm{C}$. Draw ratio $\lambda$ is 1.1 (1), 1.4 (2) 1.7 (3), 4.1 (4), 7.9 (5).

\section{Analysis of the tensile true stress-strain data}

Deformation of all the studied films is accompanied by necking, neck propagation and further homogeneous elongation of the film beyond the natural draw ratio. The sharpness of the neck is determined by the height of the yield stress in comparison to strain hardening according to Vincent's[22] this effect being more pronounced in the HDPE samples which in the neck become opaque. It should be underlined that formation of the neck starts at $\lambda-1.1-1.2$ corresponding to the yield point on the experimental stress-strain curves (Figure 9) and it ends at the natural draw ratio. Only after full propagation of the neck through the entire sample, the homogeneous deformation of the necked material proceeds into the strain hardening phase. The ultimate draw ratio reaches $\lambda=25 \div 30$.
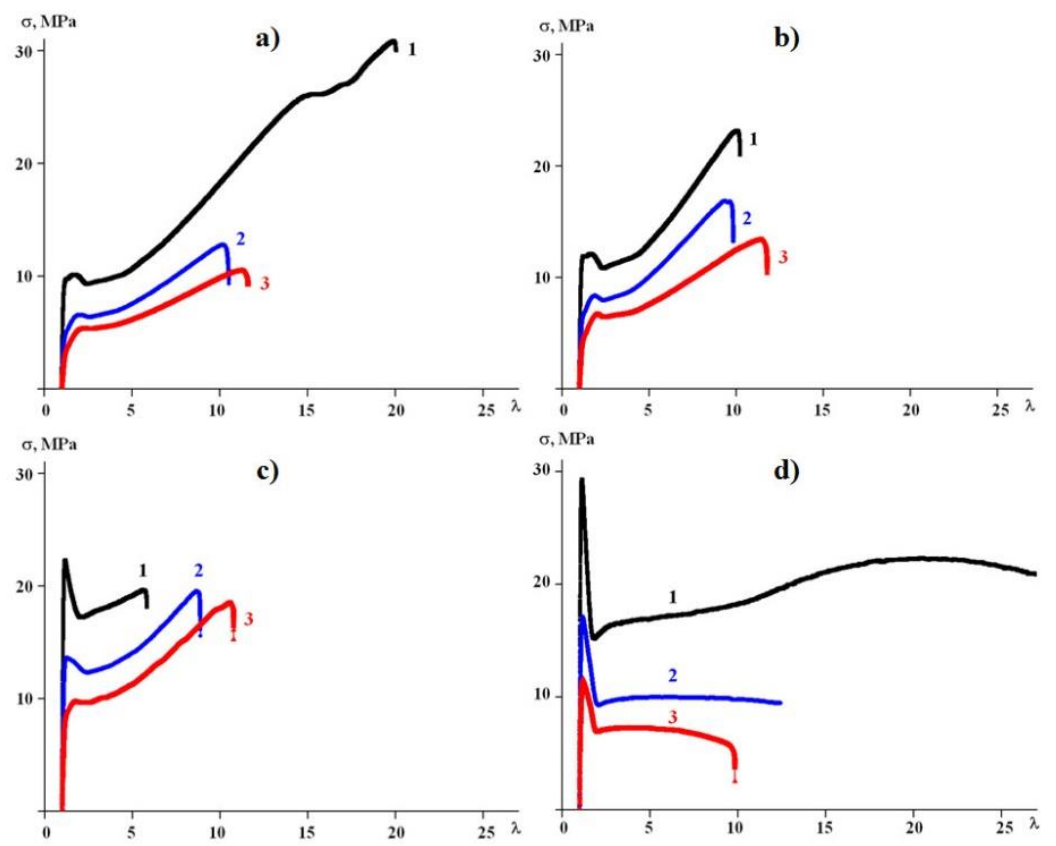

Figure 9: Experimental stress-strain curves of PE-918 (a), PE-926 (b), PE-949 (c) and PE-964 (d) drawn at $20^{\circ} \mathrm{C}(1), 50^{\circ} \mathrm{C}$ (2) and $70^{\circ} \mathrm{C}$ (3). 
Deformation of LLDPE and bimodal HDPE films appears to be more homogeneous: after its formation, the neck extends through the whole sample. In contrast to the HDPE film, both samples of LLDPE become more transparent in the neck formation, they are even more transparent than the original film very probably due to the destruction of the spherulitic-lamellar morphology and the fact that the volume change during the lamellar fibrillary transformation is less at lower crystallinities due to the reduced hydrostatic stress condition at lower density, i.e. the Poisson ratio is much closer to 0.5. This also explains why the observation is more pronounced in PE-918 than in PE-926.

The effect of temperature on the yield stress and strain hardening in polyethylene films is shown in Figure 9. Yield stress reduces with a factor two with the increase of temperature from $20^{\circ} \mathrm{C}$ to $70^{\circ} \mathrm{C}$ and also the strain hardening is reduced. Surprisingly, the strain at fracture is higher for PE-918 and PE-964 at room temperature, we attribute this to the possibility that the compression molded films of these two materials are more homogeneous than for samples with the lower melt index. The differences in the strain at fracture are less pronounced at high temperatures, which can be explained by the fact that the stresses are lower at higher temperatures and stress concentrations consequently will be lower.

The tensile curves were analyzed using rubber-elastic modelling following the Haward-Thackray assumption[15] that the dissipative component and the entropic part can be considered separately as a spring (entropy) and a dashpot (enthalpy). This approach leads to the insertion of an extra yield stress term $\sigma_{\mathrm{y}}^{*}$ to the equation:

$$
\sigma_{t r}=\sigma_{y}^{*}+\lambda \frac{\partial F}{\partial \lambda}=\sigma_{y}^{*}+G_{p}\left(\lambda^{2}-\frac{1}{\lambda}\right) \cdot \Gamma(\lambda, n)
$$

Where $G_{\mathrm{p}}=N k T$ is the strain hardening modulus as defined by Haward[23, 24,25] and $\Gamma$ takes into account the non-Gaussian chain statistics at large strains, for which Haward uses the Wang-Guth three-chain model with a Padé approximant for the inverse Langevin function[23]. For Gaussian statistics $\Gamma=1$ and (1) reduces to the well-known Neo-Hookean response.

It will be clear that such a description is only an approximation of the real mechanical response of linear short chain branched polyolefines, that might involve slipping entanglements, slipping chains and, last but not least, the network node contribution of the crystals, that our X-Ray analysis shows to be there. Only when the crystal contributions become negligible, the Haward-Thackray assumption remains valid[16]. This is actually the main reason why we performed true stress true strain measurements at $70^{\circ} \mathrm{C}$, so as to be close to the $\alpha$-relaxation of PE where the chains become mobile with respect to the crystals.

Several rubber-elastic approaches exist which incorporate or estimate the influence of entanglements, the simplest consideration coming from Mooney and Rivlin

$$
\sigma_{\text {true }}=2\left(\mathrm{C}_{1}+\frac{\mathrm{C}_{2}}{\lambda}\right)\left(\lambda^{2}-\frac{1}{\lambda}\right)
$$


This term originally had no physical meaning, but it follows naturally from developing the free energy in invariants of the strain tensor up to the first order[26,27]. Still $C_{2}$ determines a softening of the network response with increasing strain that is observed in tensile experiments that is not described by the simplest Neo Hookean description as used by Haward. This softening may be thought of as caused by a loss of entanglement density due to the deformation. The Gaussian model of Heinrich et al relates to this disentanglement as a distortion of the reptation tube[28].

Another concept that tries to capture the strain disentanglement is based on the theory of elasticity using Gaussian approximation and accounting for the topological constraints in entangled networks, it was proposed by Ball, Doi, Edwards and Warner[29,30]. In this model, the free energy of single chains between the crosslinks is described by the common statistical theory, whereas entropy reduction due to entanglements with neighboring chains is described by a number of slip-links which are free to slide along the length of an active chain between cross-links. These slip links are basically moveable network junctions that are able to slip between fixed links and hence contribute to the entropic stress built up at strain values within the strain limits of Gaussian deformation. These slipping links could be slipping entanglements as suggested by Brereton and Klein[31], but Bensasson et al.[32] have also indicated that chains slipping by crystals upon deformation may play that role which is from a physical point of view equivalent. Extra constraints introduced by such entanglements limit the extensibility of polymer macromolecules long before the limit of extensibility of single chain is reached leading to a non-Gaussian description. This effect is usually modelled by adopting Langevin statistics following Kuhn and Grün[33]. However, in this work we chose to use the description by Edwards and Vilgis[34] who extended the Gaussian Ball, Doi, Edwards and Warner model with correction for chain inextensibility:

$$
\begin{gathered}
\frac{F}{k T}=\frac{1}{2} N_{s}\left[\sum_{i=1}^{3}\left\{\frac{\lambda_{i}^{2}\left(1-\alpha^{2}\right)(1+\eta)}{\left(1-\alpha^{2} \cdot \sum_{i=1}^{3} \lambda_{i}^{2}\right)\left(1+\eta \lambda_{i}^{2}\right)}\right\}+\ln \left(1-\alpha^{2} \cdot \sum_{i=1}^{3} \lambda_{i}^{2}\right)\right] \\
+\frac{1}{2} N_{c}\left[\frac{\sum_{i=1}^{3} \lambda_{i}^{2}\left(1-\alpha^{2}\right)}{1-\alpha^{2} \cdot \sum_{i=1}^{3} \lambda_{i}^{2}}+\ln \left(1-\alpha^{2} \cdot \sum_{i=1}^{3} \lambda_{i}^{2}\right)\right]
\end{gathered}
$$

where summing is performed over the three Cartesian components of strain, $F$ is the entropic part of the free energy of the system, $N_{c}$ and $N_{s}$ are the nodal densities of cross- and slip-links correspondingly, $\lambda$ is the draw ratio of the sample. Coefficient $\eta$ which is referred to as slippage parameter, describes the freedom of slippage of the slip links. Another important coefficient $\alpha$ is an inextensibility parameter, taking into account the chain inextensibility as $\alpha=1 / \lambda_{\max }=1 / \sqrt{n_{L}}$, where $n_{L}$ is the number of Kuhn segments between fixed nodes. If $\alpha=0$, the equation is reduced to the Ball, Doi Edwards and Warner model. If $\eta=0$, slip-links are rigid and act as cross-links. Brereton and Klein have found experimentally that for PE $\eta=1.1[31]$, which implies that slip links 
may actually move beyond fixed links. This is not physically impossible when the position of fixed links themselves is allowed to relax with strain. In the course of this work we will adopt this experimental value obtained by Brereton and Klein.

Assuming the constant volume,

$$
\lambda_{1}=\lambda ; \quad \lambda_{2}=\lambda_{3}=\frac{1}{\sqrt{\lambda}}
$$

Then the stress contribution of the rubber-elastic network is obtained by the derivative of the free energy to the strain $\lambda$ at constant temperature and volume:

$$
\sigma(\lambda)=\left(\frac{\partial F(\lambda)}{\partial \lambda}\right)_{T, V}
$$

Following Haward and Thackray's assumption the dissipative contribution to the stress is captured in yield stress like contribution $\sigma_{y}^{*}(T, \dot{\varepsilon})$ :

$$
\sigma=\sigma_{y}^{*}(T, \dot{\varepsilon})+\left(\frac{\partial F(\lambda)}{\partial \lambda}\right)_{T, V}
$$

One has thereby always to keep in mind that the Haward-Thackray assumption is an in principle incorrect simplification and that the strain hardening contribution to stress is only partial entropic. This is reflected in our measurements because increasing the temperature leads to lower node densities $N_{c}$ and $N_{s}[10]$. For a typical rubber with fixed links an opposite would be true, the well-known characteristics of reversible rubber elasticity including the proportionality of stress to absolute temperature.

Including the yield stress, the slip-link model features four independent parameters if we assume $\eta=1.1$ and it is able to describe the whole stress-strain dependence. The initial portion of stress-strain curves reveals the typical double yield points. In regular HDPE (PE-964) the stress of the first yield point is higher than that of the second one, while in other samples the situation is opposite. With the increase of deformation temperature, the first yield stress decreases more significantly. The double yield feature disappears when mapped in true stress true strain as it was observed by Strobl et al.[35], who concluded that the double yield feature is an artefact of the nominal stress strain curve. However true stress strain compression tests also reveal the double yield feature, indicating that this phenomenon is intrinsic to the deformation process and is not an artefact. Kanters[36] also observed that the first yield point decreases faster with lowering the strain rate, which is in accord with our observation of temperature sensitivity of the first yield point.

Seguela and Rietsch[37] also observe this temperature sensitivity, and they explain the double yield observed as two different stages lamellae, a collective lamellar shear slip followed by fragmentation.

The density dependencies of the mechanical parameters of the studied samples obtained from the experimental stress-strain curves at $70^{\circ} \mathrm{C}$ are presented on Figure 10. In general, it is expected that an increase of the density of branched polyethylene leads to a substantial increase of the fracture stress and to a decrease of the strain at fracture. The ongoing increase in fracture 
stress with density is not observed for the PE-964 sample which may be attributed to the high MFI and a lower availability of load bearing molecules.
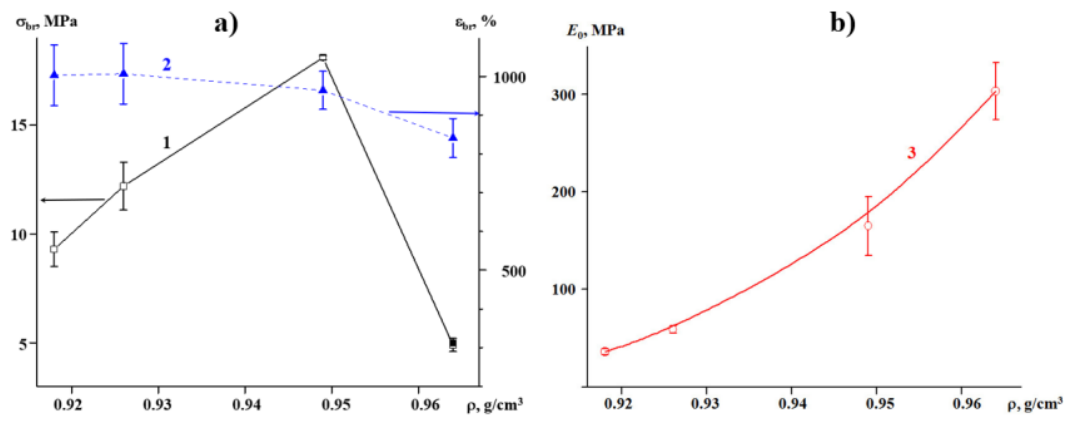

Figure 10: Density dependence of the mechanical parameters of the studied PE samples at $70^{\circ} \mathrm{C}$ : breaking stress (1), breaking strain (2), Young modulus (3).
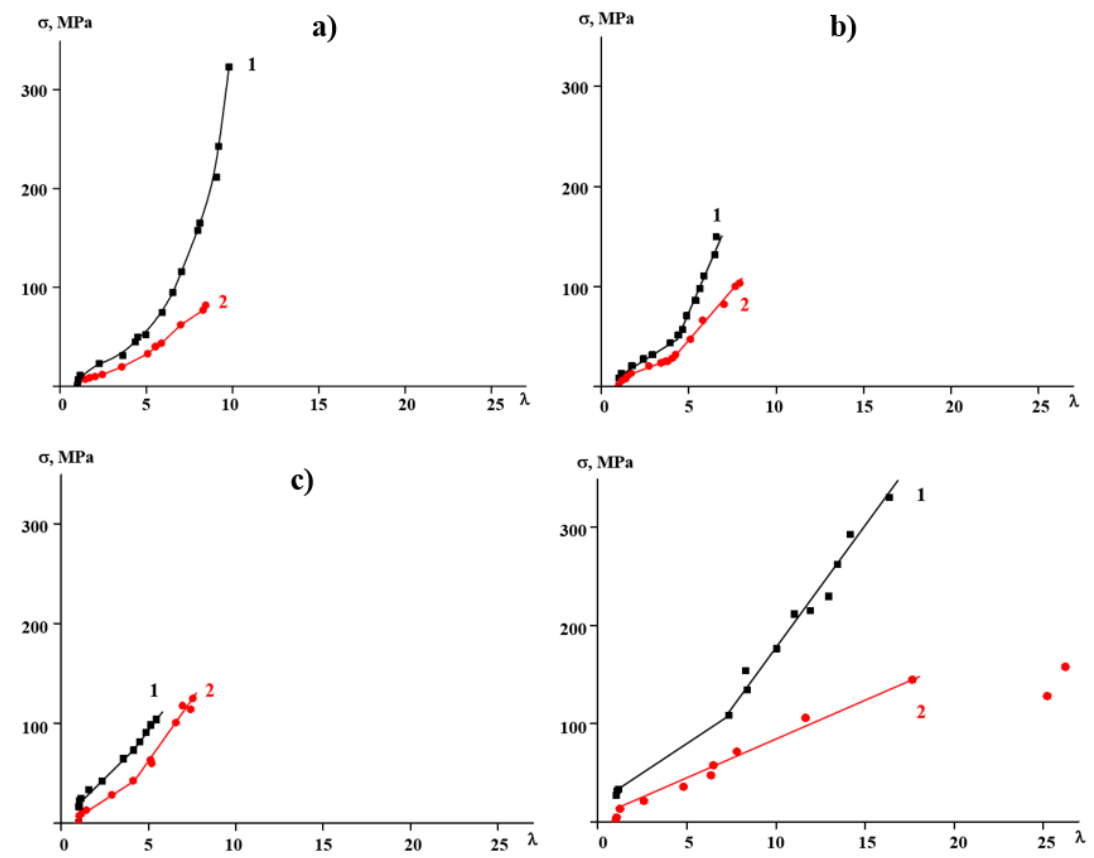

Figure 1l: True stress-strain curves of PE-918 (a), PE-926 (b), PE-949 (c) and PE-964 (d) drawn at $20^{\circ} \mathrm{C}(\mathrm{l})$ and $70^{\circ} \mathrm{C} \mathrm{(2)}$.

Analysis of digital images of the oriented samples allows reconstructing the true stress - true strain curves using the values of local strains estimated from the deformation of the ink grid (Figure ll). This approach allows for an accurate interpretation of structural changes observed by X-Ray scattering and described below. Comparison of experimental and true stress-strain curves for all PE samples drawn at 20 and $70^{\circ} \mathrm{C}$ reveals a number of significant differences: the yield point on true stress-strain 
dependencies appears as a downward change of slope in all curves, the second deviation point corresponds to the onset of strain hardening, the natural draw ratio. This second tangent is not so distinct for HDPE, because of its high MFI. The stress of deformation at $T_{\text {def }}=70^{\circ} \mathrm{C}$ is lower in the whole range of strains $\lambda$, and the true fracture strain is always higher at $T_{\text {def }}=70^{\circ} \mathrm{C}$. The comparatively high true fracture stress of low crystalline PE-918 at room temperature is easily explained by the high tie molecular content[38] but also the relative insensitivity to stress concentrations due to the low yield stress.

It should also be noted that the strain hardening part of all the true stress-strain curves at $70^{\circ} \mathrm{C}$ but the PE-964 samples can be approximated by a straight line in $\lambda$. Its slope is inversely proportional to the MFI of PE: 0.14, 0.19 and 0.26 for PE-918, PE-926 and PE-949 correspondingly. This straight line in $\lambda$ is indicative of a network response with vanishing and/or slipping nodes[10]. Decrease of the yield stress with temperature is negligibly small for LLDPE (PE-918 and PE-926) samples compared to that in HDPE ones.
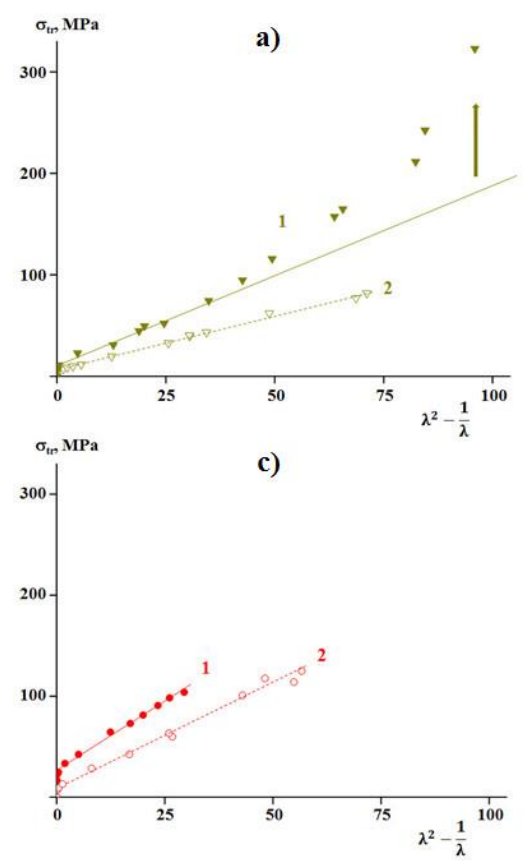
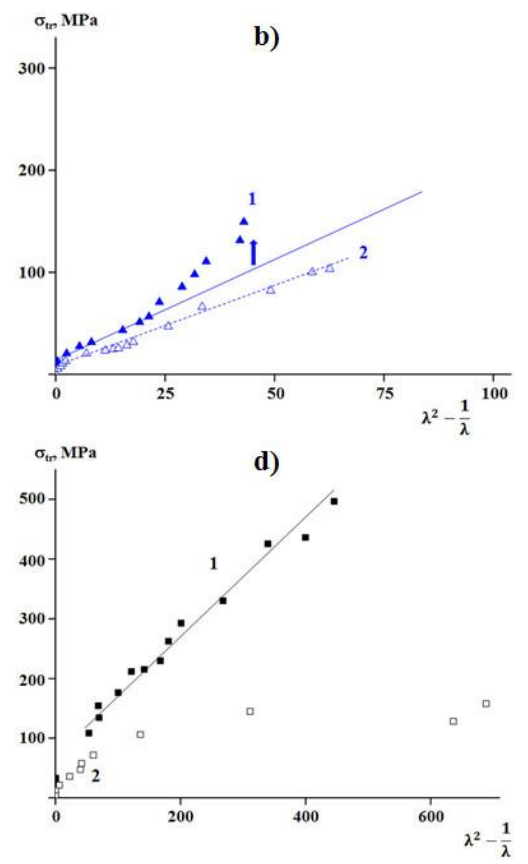

Figure 12: Haward plots of the orientation of PE-918 (a), PE-926 (b), PE-949 (c) and PE-964 (d) drawn at $20^{\circ} \mathrm{C}$ (1) and $70^{\circ} \mathrm{C} \mathrm{(2).} \mathrm{Non-Gaussian}$ upswings of the plots a (l) and b (l) are shown by the arrows.

Haward plots of the true stress as a function of the neohookean true strain measure, of all PE samples stretched at Tdef $=70^{\circ} \mathrm{C}$ feature a straight line in the strain hardening region of the deformation. (Figure 12). It must be emphasized here that a straight line in a neohookean plot beyond $\lambda=3$ means that Gaussian statistics is actually obeyed beyond the physically valid range[33]. This can only be when nodes vanish as a function of strain, i.e. an ongoing disentanglement with strain. A net upswing in stress at higher strains 
was observed for both LLDPE materials at room temperature. This indicates that there are less to none vanishing nodes and it may also indicate that nongaussian chain segment statistics, i.e inextensibility, kicks in. The values of the strain hardening modulus $G_{\mathrm{p}}$ and of the molecular mass of the internode chain segments $M_{\mathrm{cs}}$ obtained from the plot fits using Haward approach are summarized in Table 2. These results appear to be physically plausible as they are in the range of the plateau modulus of polyethylene i.e. $1.5 \mathrm{MPa}$ $\left(M_{\mathrm{e}}=1220 \mathrm{~g} / \mathrm{mole}\right)$. The tendency for the increase of $M_{\mathrm{cs}}$ with decreasing density can be explained by the decreased available entanglements density due to a decrease in average molecular mass. Haward has concluded that although these values are of the same order of magnitude, they need not be identical[39]. There must indeed be a contribution from the crystalline phase to the stress built up in the strain hardening part, notwithstanding the fact that the original crystals have yielded at far lower true stresses. This is exactly where the Haward-Thackray assumption loses its validity and measurements at temperatures close to the $\alpha$ transition are needed to assess the available molecular network.

The observed non-Gaussian upswing of Haward plots for the deformation of PE-918 and PE-926 at room temperature are to be attributed to the strain hardening caused by a limited extensibility of the network chain segments. That such strain hardening does not occur for HDPE is due to intensive processes of slippage caused by insufficient molecular mass to prevent tie molecules from slippages[38]. As we will see further, there is not an a priori reason to assume an increase in the number of network junctions with strain (such as stress induced crystallization) because no upswing beyond the normal rubber elastic response occurs, although it cannot be excluded that there is a dynamic balance between vanishing and created nodes with increasing strain. It is clear that the stress level is always higher at room temperature compared to that at $70^{\circ} \mathrm{C}$. This difference is comparatively small for bimodal PE-949 as well as for PE-964. This stress drop with temperature in the strain hardening phase is a strong indication that the network response is not entropic in nature.

Application of the Mooney-Rivlin approach confirms the results obtained from the neohookean analysis. The value of $\mathrm{Cl}$ (equation 2) obtained from the value of stress at this plateau allows an alternative estimation of the shear modulus associated with strain hardening.

Application of the slip link model starts with evaluating the data up to $\lambda=5$ using the Ball, Doi, Edwards and Warner model. Because above the yield stress and below the Gaussian draw ratio limit only a very limited data set is available and because the equation to be fitted contains four variables and in the non Gaussian case even five (see next section) we will attempt to make sound physical estimates of most parameters. Moreover equation (3), when used as a purely mathematical fit function easily converges to subminima without any physical meaning, so a wise fit strategy is required.

The initial yield stress $\sigma^{*}{ }_{\mathrm{y}}$ is estimated from the Neo Hookean analysis. The starting value for the fixed network density is also taken from the Neo 
Hookean analysis and the slip link contribution to the network modulus is initially set to 0 . The slippage freedom parameter $\eta$ is set fixed at a value of 1.1 following Brereton and Klein's experimental value for PE. Then the dataset is fitted with the Ball, Doi Edwards and Warner to obtain the slip link contribution $G_{\mathrm{s}}$ and the fixed link contribution $G_{\mathrm{c}}$ to the stress. For a network without slip links; $G_{\mathrm{s}}=0$ and $G_{\mathrm{c}}=G_{\mathrm{p}}$. When slip links are present there is no simple direct relation between $G_{\mathrm{p}}$ and $G_{\mathrm{c}}$ but as an order of magnitude $G_{\mathrm{p}} \approx G_{\mathrm{c}}+0.1 \cdot G_{\mathrm{s}}$ should hold. In a second step the parameters obtained from the BDEW model serve as starting values for an optimization of the inextensibility parameter $\alpha$ in the Edwards-Vilgis model. Table 2 summarizes the mechanical parameters obtained from all the models applied to the true stress-strain curves of the studied PE samples with different densities.
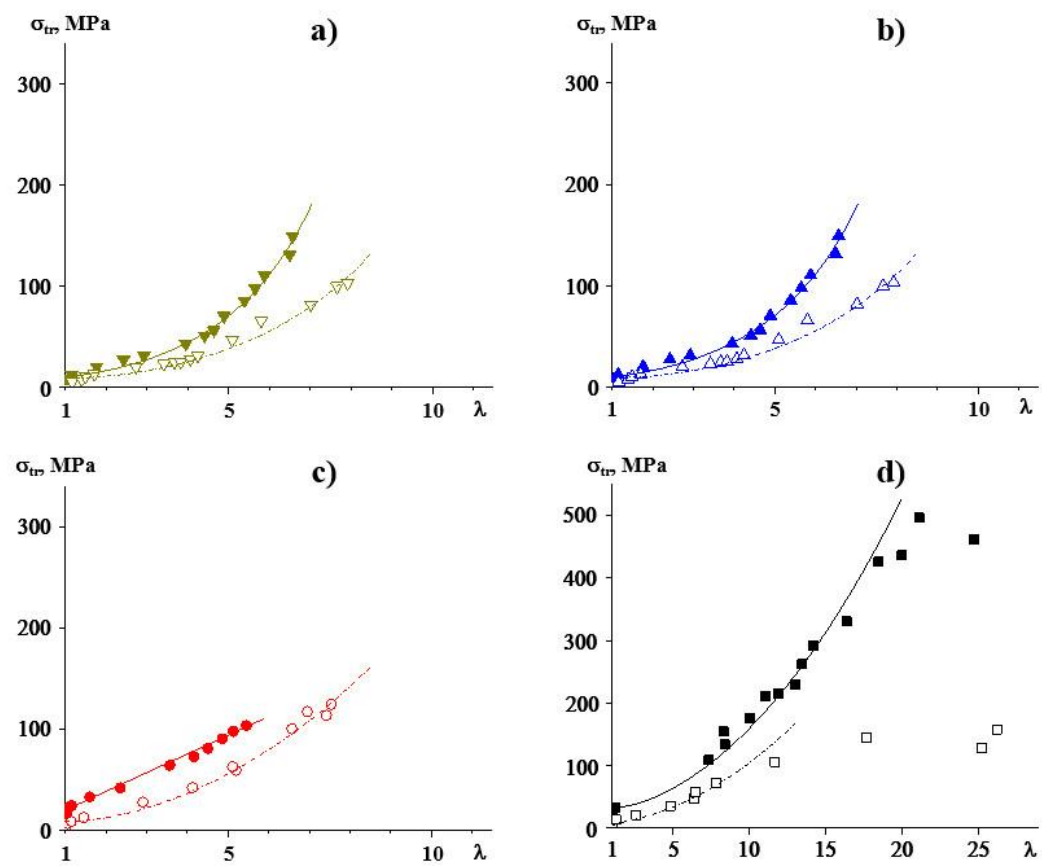

Figure 13: Application of the slip-link model to the orientation of PE-918 (a), PE-926 (b), PE-949 (c) and PE-964 (d) drawn at $20^{\circ} \mathrm{C}$ (l) and 70 $\mathrm{C}$ (2). 
Table 2. Mechanical characteristics of the studied materials obtained from the application of different models

\begin{tabular}{|c|c|c|c|c|c|c|c|c|}
\hline \multirow{2}{*}{ Sample } & \multirow{2}{*}{$\mathrm{T},{ }^{\circ} \mathrm{C}$} & \multicolumn{2}{|c|}{ Haward - Thackray } & \multicolumn{2}{|c|}{ Mooney - Rivlin } & \multicolumn{3}{|c|}{ Slip-link analysis } \\
\hline & & $G_{\mathrm{p}}, \mathrm{MPa}$ & $M_{\mathrm{cs}}, \mathrm{g} /$ mole & $G_{p}, \mathrm{MPa}$ & $\mathrm{M}_{\mathrm{cs}}, \mathrm{g} / \mathrm{mol}$ & $\mathrm{G}_{\mathrm{c}}, \mathrm{MPa}$ & $\mathrm{G}_{\mathrm{s}}, \mathrm{MPa}$ & $\alpha^{-1}$ \\
\hline \multirow{2}{*}{ PE-918 } & 20 & 1.8 & 1400 & 2.4 & 1034 & 1.4 & 18.0 & 18 \\
\hline & 70 & 1.1 & 2900 & 1.4 & 1714 & 1.1 & 3.1 & 71 \\
\hline \multirow{2}{*}{ PE-926 } & 20 & 2.1 & 1100 & 2.8 & 887 & 1.6 & 18.0 & 13 \\
\hline & 70 & 1.5 & 2062 & 1.8 & 1714 & 1.1 & 7.3 & 19 \\
\hline \multirow{2}{*}{ PE-949 } & 20 & 2.8 & 875 & 3.1 & 804 & 2.5 & 11.1 & - \\
\hline & 70 & 2.1 & 1580 & 2.3 & 1329 & 2.1 & 2.5 & 56 \\
\hline \multirow{2}{*}{ PE-964 } & 20 & 1.3 & 1934 & 1.3 & 1923 & 1.5 & 0.0 & 333 \\
\hline & 70 & 1.0 & 2750 & 1.0 & 3060 & 0.9 & 19.3 & - \\
\hline
\end{tabular}


Separation between fixed and slip link network contributions reveals neatly that although there is a slight drop in the fixed link network density with increasing temperature, this contribution remains of the same order of magnitude and predominantly relates to the molecular mass. This observation supports the idea that the network of entanglements that remain fixed on the time scale of the measurement is actually entropic in nature and does not depend greatly on temperature. Considering the slip link contributions, one can find them to be an order of magnitude larger than the fixed links. This matches the idea that it takes about 10 entanglements to fix one chain segment on the time scale of our tensile experiment. We expect that upon decrease of the strain rate, this number will be larger. The typical dimensions of chain segment molecular masses involved in slip link activity are of the order of 250 to $300 \mathrm{~g} / \mathrm{mole}(-10 \mathrm{MPa}$ ) amounting to about 20 monomer units. Taking into account the Kuhn segment ( $100 \mathrm{~g} /$ mole), the length of short chain branched PE of approximately $1 \mathrm{~nm}$ corresponds to about three Kuhn segments per slip link. This is comparable to the reptation tube diameter which is of the order of 5-10 nm and to the thickness of interlamellae amorphous layers in PE (An apparent tube diameter in HDPE melts, i.e., $\mathrm{d}=6$ and $4.87 \mathrm{~nm}$ for PE with $\mathrm{Mw}=12.400$ and $190.000 \mathrm{~g} / \mathrm{mol}$, respectively [40]. Other studies reported tube diameter in the range from 3.2 to $4.5 \mathrm{~nm}$ [41]).

). More specifically the slip link network density appears to decrease with the amount of short chain branching. This would appear to bear a relation with the slip hindrance caused by short chain branches. Presumably the contour and the span distance along the main chain (crystallisable sequences) are responsible for this increased hindrance.

The slip link model also explains why the least branched molecule i.e. HDPE-964 features an increase in slip link contribution with increasing temperature: with long crystallisable sequences the oncoming thermally activated mobility will engender more and more slip possibilities. As soon as this attachment-detachment processes become hindered by short chain branches the activity of slip links remains limited to ambient temperature but becomes fixed by the molecular 'hooks' in the crystals.

So, we gained insight into the physical nature of the network of poyoleffines in a wide range of their crystallinity. It turns out that the highest network density at high temperatures (indicative for long time response) requires both a high molecular mass and short chain branches and that solely crystallinity is not sufficient to generate strain hardening. This conclusion supports the important role of effective tie molecules in such a network.

\section{Summary}

The present investigation of various PE films shows that the samples are microheterogeneous semicrystalline material. Their crystal structure exhibits a size scale of tens of nm. Initial morphology includes the lamellar structure with different degree of continuous crystalline network formation. The true stress-strain curves were measured. Despite similarity in the curves they reveal the different regions of deformation and of transition from the original films to uniaxially oriented samples. 
In order to better understand the structure-property relationship the deformation mechanism of samples was studied by DSC and X-ray. It was established that the transformation from original lamellar to final fibrillar structure passes through the similar stages: deformation of initial lamellae ( $\varepsilon<50 \%$ ); destruction of lamellar structure trough the tilt, slippage of molecules in crystallites, their twining, which takes place in necking area; simultaneous formation of fibrils with structural characteristics depending on molecular structure and on deformation conditions; deformation of formed fibrillar structure, tilting - formation of chevron for high MM polymer (LLDPE) or slippage of fibrils and void formation. The density of amorphous regions increases as well as a number of tie molecules leading to rise of mechanical properties.

Slip-link model was proved to be applicable to such partially crystalline materials despite of the apparent energy effects of deformation, giving feasible estimation of the molecular processes during orientation.

\section{Aknowledgements}

This work was supported by Russian Science Foundation (project 14-1301402).

\section{References}

[1] N. R. Legge, G. Holden, H. E. Schroeder, Thermoplastic Elastomers, Hanser Publishers, 1996.

[2] J. G. Van Alsten, B. B. Sauer, C. R. Gochanour, K. L. Faron, W. D. Dozier, Interfacial Broadening and Self-Adhesion of Segmented Block Copolymers Studied by Neutron Reflection, Macromolecules 28 (20) (1995) 7019-7021. doi: 10.1021/ma00124a045.

[3] Yu. K. Godovsky, E. V. Konyukhova, S. N. Chvalun, V. M. Neverov, A. S. Abu-Surrah, B. Rieger, Stretching calorimetry and X-ray characterization of deformational behavior of new high molecular weight propene-carbon monoxide alternating co- and terpolymers, Macromol. Chem. Phys. 200 (1999) 2636-2644. doi: 10.1002/(SICI)15213935(19991201)200:12く2636::AID-MACP2636〉3.0.CO;2-\#

[4] U. Dietrich, M. Hackmann, B. Rieger, M. Klinga, M. Leskelä, Control of Stereoerror Formation with High-Activity "Dual-Side" Zirconocene Catalysts: A Novel Strategy To Design the Properties of Thermoplastic Elastic Polypropenes, J. Am. Chem. Soc. 121 (1999) 4348-4355. doi: 10.1021/ja9833220.

[5] K. P. Gallagher, X. Zhang, J. P. Runt, G. Huynh-ba, J. S. Lin, Miscibility and cocrystallization in homopolymer-segmented block copolymer blends, Macromolecules 26 (4) (1993) 588-596. doi: 10.1021/ma00056a006.

[6] B. Rieger, C. Troll, J. Preuschen, Ultrahigh Molecular Weight Polypropene Elastomers by High Activity "Dual-Side" Hafnocene Catalysts, Macromolecules 35 (15) (2002) 5742-5743. doi: 10.1021/ma025520o.

[7] J. Voegele, C. Troll, B. Rieger, Zirconocene-catalyzed propene-ethene copolymer elastomers: Kinetic investigations at low ethene concentration 
and characterization of microstructure, Macromol. Chem. Phys.203 (13) (2002) 1918-1925. doi: 10.1002/1521-3935(200209)203:13<1918::AIDMACP1918>3.0.CO;2-4

[8] A. Schmidt, W. S. Veeman, V. M. Litvinov, W. Gabrielse, NMR Investigations of In-Situ Stretched Block Copolymers of Poly(butylene terephthalate) and Poly(tetramethylene oxide), Macromolecules 31 (5) (1998) 1652-1660. doi: 10.1021/ma9714676.

[9] M. A. Shcherbina, M. Yu. Meshchankina, Ya. I. Odarchenko, M. Machat, B. Rieger, S. N. Chvalun, From Elastomers to Thermoplasts - Precise Control of Isotactic Propylene Structure and Properties and the Role of Different Structural Elements in its Mechanical Behaviour, Polymer. 113 (2017) 213222. DOI: 10.1016/j.polymer.2017.11.038.

[10] R. A. C. Deblieck, D. J. M. Van Beek, K. Remerie, I. M. Ward, Failure mechanisms in polyolefines: The role of crazing, shear yielding and the entanglement network, Polymer 52 (2011) 2979-2990. doi:10.1016/j.polymer.

[11] E. J. Kramer, L. L. Berger, Fundamental processes of craze growth and fracture, Adv. Polym. Sci. 91/92 (1990) 1-68. doi: 10.1007/BFb0018018.

[12] S. Radhakrishnan, D. R. Saini, M. V. Kuber, Effect of Morphology on the dielectric properties of a segmented copolyester, Eur. Polym. J. 27 (3) (1991) 291-297. doi: 10.1016/0014-3057(91)90108-Z.

[13] A. M. Donald, E. J. Kramer, Effect of molecular entanglements on craze microstructure in glassy polymers, J. Polym. Sci. Phys. Ed. 20 (5) (1982) 899-909. doi: 10.1002/pol.1982.180200512.

[14] J. J. Mckechnie, R. N. Haward, D. Brown, J. H. R. Clarke, Effects of chain configurational properties on the stress-strain behavior of glassy linear polymers, Macromolecules 26 (l) (1993) 198-202. doi: 10.1021/ma00053a030.

[15] R. N. Haward, G. Thackray, The Use of a Mathematical Model to Describe Isothermal Stress-Strain Curves in Glassy Thermoplastics, Proc. R. Soc. Lond. A 302 (1968) 453-472. doi: 10.1098/rspa.1968.0029.

[16] N. P. Bessonova, S. V. Krasheninnikov, Ya. I. Odarchenko, The contribution of various network knots during the deformation of ethylene copolymers and nanocomposites based on them, Polym. Sci. A. 58 (4) (2016) 556-566.

[17] S. N. Chvalun, A. N. Ozerin, Yu. A. Zubov, Yu. K. Godovsky, Calorimetric studies of the process of elastic loading of linear polyethylene oriented films with different structure of amorphous regions, VYSOKOMOLEKULYARNYE SOEDINENIYA SERIYA B 20 (9) (1978) 672-674.

[18] A. N. Ozerin, Yu. A. Zubov, V. I. Selikhova, S. N. Chvalun, N.F. Bakeev, Use of the method of measuring the absolute intensity of small angle $X$ Ray scattering for the study of the structure of amorphous regions in oriented polyethylene films, Polymer Science U.S.S.R., 18 (9) (1976) 2434 2442.

[19] A. N. Ozerin, Yu. A. Zubov, S.N. Chvalun, N. F. Bakeev, G. P. Belov, X-Ray structural analysis of amorphous regions in oriented films of linear polyethylene, Polymer Science U.S.S.R., 19 (5) (1977) 1223-1231.

[20] M. B. Konstantinopolskaya, S. N. Chvalun, V. I. Selikhova, Structure of high-oriented polyethylene samples obtained by uniaxial stretching of monocrystal mats, VYSOKOMOLEKULYARNYE SOEDINENIYA SERIYA B 27 (7) (1985) 538-541.

[21] A. Peterlin, Drawing and extrusion of semi-crystalline polymers, Colloid Polym. Sci., 265 (5) (1987) 357-382. doi: 10.1007/BF01412215. 
[22] P. I. Vincent, The necking and cold-drawing of rigid plastics, Polymer 1 (1960) 7-19.

[23] P. J. Mills, J. N. Hay, R. N. Haward, Analysis of the rubber elasticity of polyethylene networks on the slip-link model of S. F. Edwards et al., J. Mater. Sci. 20501 (1988) 970-974. doi: 10.1016/0032-3861(88)90002-X.

[24] R. N. Haward, Strain hardening of thermoplastics, Macromolecules 26 (22) (1993) 5860-5869. doi: 10.1021/ma00074a006.

[25] R. N. Haward, The application of non-Gaussian chain statistics to ultralow density polyethylenes and other thermoplastic elastomers, Polymer 40 (21) (1999) 5821-5832. doi: 10.1016/S0032-3861(99)00124-X.

[26] M. Mooney, A theory of large elastic deformation, J. Appl. Phys. 11 (1940) 582-592. doi: 10.1063/1.1712836.

[27] R. S. Rivlin, Large Elastic Deformations of Isotropic Materials. IV. Further Developments of the General Theory, Phil. Trans. R. Soc. A24l (1948). 379397. doi: 10.1098/rsta.1948.0024.

[28] G. Heinrich, E. Straube, G. Helmis, Rebber elasticity of polymer networks: Theories, Adv. Polym. Sci. 85 (1988) 33-87. doi:10.1007/BFb0024050.

[29] R. C. Ball, M. Doi, S. F. Edwards, M. Warner, Elasticity of entangled networks, Polymer 22 (8) (1981) 1010-1018. doi: 10.1016/00323861(81)90284-6.

[30] P. Thirion, T. Weil, Assessment of the sliding link model of chain entanglement in polymer networks, Polymer 25 (5) (1984) 609-614. doi: 10.1016/0032-3861(84)90027-2.

[31] M. G. Brereton, P. G. Klein, Analysis of the rubber elasticity of polyethylene networks based on the slip link model of S. F. Edwards et al., Polymer 29 (8) (1988) 970-974. doi: 10.1016/0032-3861(88)90002-X.

[32] S. Bensason, E. V. Stepanov, S. Chum, A. Hiltner, E. Baer, Deformation of elastomeric Ethylene-Octene Copolymers, Macromolecules 30 (1997) 2436-2444. doi: 10.1021/ma961685j.

[33] W. Kuhn, F. Grün. Kolloid Z. 101 (1942) 248.

[34] E S. F. Edwards, Th. Vilgis, The effect of entanglements in rubber elasticity, Polymer 27 (4) (1986) 483-492. doi: 10.1016/00323861(86)90231-4.

[35] S. Hobeika, Y. Men, G. Strobl, Temperature and Strain Rate Independence of Critical Strains in Polyethylene and Poly(ethylene-covinyl acetate), Macromolecules 33 (2000) 1827-1833. doi: 10.1021/ma9910484.

[36] M. Kanters, Prediction of Long-Term Performance of Load-Bearing Thermoplastics, PhD Thesis University Eindhoven 2015.

[37] R. Seguela, F. Rietsch, Double yield points in polyethylene under tensile loading, J. Mater. Sci. Lett. 9 (1990) 46-47. doi: 10.1002/pen.760351303.

[38] R. Seguela, Critical review of the molecular topology of semicrystalline polymers: The origin and assessment of intercrystalline tie molecules and chain entanglements, J. Polym. Sci. Part B: Polym. Phys. 43 (2005) 17291748. doi: 10.1002/polb.20414.

[39] R. N. Haward, Strain hardening of high density polyethylene, J. Polym. Sci. Part B: Polym. Phys. 45 (2007) 1090-1099. doi: 10.1002/polb.21123.

[40] A. Wischnewski, M. Monkenbusch, L. Willner, D. Richter, A. E. Likhtman, T. C. B. McLeish, B. Farago, Molecular observation of contourlength fluctuations limiting topological confinement in polymer melts. Phys. Rev. Lett. 88 (2002) 058301-11. 
[41] K. Moorthi, K. Kamio, J. Ramos, D. N. Theodorou, Monte Carlo simulation of short chain branched polyolefins: Structure and properties. Macromolecules 45 (2012) 8453-8466. 